\title{
The Utility of the Alvarado Score in the Diagnosis of Acute Appendicitis in the Elderly
}

ANASTASIYA SHCHATSKO, M.D., ${ }^{*}$ REBECCA BROWN, M.D., ${ }^{*}$ TRISTA REID, M.D., ${ }^{*}$ SASHA ADAMS, M.D.,† AMY ALGER, M.D.,‡ ANTHONY CHARLES, M.D., M.P.H.*

*From the Department of Surgery, University of North Carolina at Chapel Hill, Chapel Hill, North Carolina; tDepartment of Surgery, University of Texas, Houston, Texas; and $\ddagger$ Department of Surgery, Duke University,

Durham, North Carolina

\begin{abstract}
Clinical scores determining the likelihood of acute appendicitis (AA), including the Alvarado score, were devised using a younger population, and their efficacy in predicting AA in elderly patients is not well documented. This study's purpose is to evaluate the utility of Alvarado scores in this population. A retrospective chart review of patients $>65$ years old presenting with pathologically diagnosed AA from 2000 to 2010 was performed. Ninety-six patients met inclusion criteria. The average age was $73.7 \pm 1.5$ years and our cohort was 41.7 per cent male. The average Alvarado score was $6.9 \pm 0.33$. The distribution of scores was 1 to 4 in 3.7 per cent, 5 to 6 in 37.8 per cent, and 7 to 10 in 58.5 per cent of cases. There was a statistically significant increase in patients scoring 5 or 6 in our cohort versus the original Alvarado cohort $(P<0.01)$. Right lower quadrant tenderness $(97.6 \%)$, left shift of neutrophils $(91.5 \%)$, and leukocytosis $(84.1 \%)$ were the most common symptoms on presentation. In conclusion, our data suggest that altering our interpretation of the Alvarado score to classify elderly patients presenting with a score of $\geq 5$ as high risk may lead to earlier diagnosis of AA. Physicians should have a higher clinical suspicion of AA in elderly patients presenting with right lower quadrant tenderness, left shift, or leukocytosis.
\end{abstract}

A CUTE APPENDICITIS Is the most common cause of abdominal pain resulting in surgery in the United States, with approximately seven per cent of the American population experiencing appendicitis in their lifetime. The diagnosis and management of this disease have evolved with continued medical and surgical advancements. Appendicitis is known to be a disease of younger age groups with only 5 to 10 per cent of cases occurring in the elderly population. ${ }^{1}$ However, the incidence of the disease in older age group seems to be rising, likely due to increase in life expectancy. 1,2

Acute appendicitis in the elderly is often characterized by an atypical presentation and a delay in seeking medical attention. The prognosis of uncomplicated appendicitis in both young and old age groups is nearly equal. ${ }^{3}$ However, the delay in presentation, diagnosis, and treatment in the elderly results in worsened clinical condition at presentation and higher perforation rates leading to increased morbidity and mortality. ${ }^{3}$

Presented as an ePoster at the Southeastern Surgical Congress 2017 Annual Scientific Meeting, February 27, 2017, Nashville, TN.

Address correspondence and reprint requests to Anthony Charles, M.D., M.P.H., Department of Surgery, University of North Carolina School of Medicine, 4008 Burnett-Womack Building, CB 7228, Chapel Hill, NC 27599. E-mail: anthchar@med.unc.edu.
Several scoring systems for determining the likelihood of acute appendicitis based on presenting signs, symptoms, and laboratory values have been developed and used clinically for many years. One such score, the Alvarado score, ${ }^{4}$ was developed based on the presentation pattern, clinical and laboratory variables of a younger population (mean patient 23.4-25.9 years of age $)^{4,5}$ and was validated in this younger cohort. However, the utility, reliability, and accuracy of these scoring systems in predicting acute appendicitis in patients 65 years of age and older is unknown. We therefore sought to evaluate the utility of the Alvarado score in predicting acute appendicitis in the elderly.

\section{Methods}

\section{Study Design}

This is a retrospective review of a Department of Pathology database of all surgical specimens containing the vermiform appendix from January 2000 to December 2010 at the University of North Carolina. A subset of elderly patients ( $\geq 65$ years of age) who presented consecutively with appendicitis based on pathologic criteria and report were selected manually from the database. Patients with a pathologic diagnosis of chronic appendicitis, those who presented for elective interval 
appendectomy, or those who had incidental findings of appendicitis during other procedures, were excluded.

For patients meeting inclusion criteria, individual medical records were reviewed. Data points that were extracted included baseline demographics, such as age and sex, as well as clinical and laboratory variables, including time from onset of pain to hospital presentation, migration of pain to the right lower quadrant (RLQ), presence of anorexia, nausea or vomiting, body temperature, white blood cell (WBC) level, left shift or neutrophilia $\geq 70$ per cent, tenderness in the RLQ, rebound tenderness, previous emergency department or clinic visits, and time from symptoms to operative intervention. Outcome variables included hospital length of stay, pathological characteristics of the vermiform appendix, postoperative complications, and discharge destination. These data were then used to calculate an Alvarado score for each patient. Data variables and components of the Alvarado score are outlined in Table 1. The standard interpretation of the Alvarado score is that scores of 1 to 4 correspond to low, 5 to 6 to intermediate, and 7 to 10 to high risk for appendicitis.

\section{Data Analysis}

Presenting symptoms, occurrence of different pathological forms of appendicitis [categorized as uncomplicated (nonperforated or suppurative) and complicated (perforated or gangrenous) appendicitis], and Alvarado scores for each patient were calculated. We then compared the resulting score distribution of our data set with the original Alvarado data. ${ }^{4}$

Categorical variables were analyzed with the chisquared test, whereas continuous variables were analyzed by the $t$ test. The Wilcoxon rank sum test was used for comparison of abnormally distributed groups of data, and $P<0.05$ were considered statistically significant.

\section{Results}

A total of 114 patients $>65$ years of age were identified in the database, and 96 patients with pathologically proven

TABLE 1. The Alvarado Scoring System ${ }^{4}$

\begin{tabular}{lc}
\hline \multicolumn{1}{c}{ Variable } & Score \\
\hline WBC count $\geq 10,000$ & 2 \\
RLQ tenderness & 2 \\
Pain migration & 1 \\
Rebound pain & 1 \\
Temperature $\geq 37.3^{\circ} \mathrm{C}$ & 1 \\
Nausea or vomiting & 1 \\
Anorexia & 1 \\
Left shift of neutrophils (neutrophils $\geq 70 \%)$ & 1 \\
Total & 10 \\
\hline
\end{tabular}

acute appendicitis met inclusion criteria. The mean age was $73.7 \pm 1.5$ years (range: $65-104$ ) and the study population was 41.7 per cent male (40/96), with the male to female ratio 1:1.4.

\section{Preadmission Variables}

The mean duration of pain before presentation was $2.8 \pm 0.8$ days. The duration of pain before presentation to the emergency room (ER) was 1.4 days for patients with uncomplicated appendicitis and 3.9 days for patients with complicated appendicitis $(P=$ $0.0003)$. Delayed presentation to the hospital of $\geq 48$ hours was found in 42 out of 91 patients $(46.2 \%) ; 5$ patients were excluded from this analysis due to lack of appropriate data. A delay in presentation was observed in $10(25 \%)$ and $32(62.7 \%)$ cases of uncomplicated and complicated appendicitis, respectively. A total of $10(11.6 \%)$ patients had been seen in a nearby ER or in outpatient clinic and were diagnosed with diverticulitis or urinary tract infection before presentation to University of North Carolina; all of these patients represented and were appropriately diagnosed with delay of $9.2 \pm 5.6$ days from the onset of symptoms. All were subsequently found to have complicated appendicitis.

\section{Diagnostic Variables}

CT was performed in 94 (97.9\%) cases. In two cases, CTs were performed twice, and in one case three CT scans were obtained. CT eventually led to the correct diagnosis in only one of the two cases. Acute appendicitis was correctly diagnosed in $80(81.6 \%)$ of completed CTs and missed in $5(5.1 \%)$. Incorrect diagnoses included indefinite diagnosis or an unrelated disease diagnosed in 13 (13.3\%) CT scans. Ultrasound was performed in seven $(7.3 \%)$ cases with negative result in three and indeterminate result in four studies.

\section{Preoperative Course}

The mean time between presentation to the ER and transfer to the operating room was $10.3 \pm 2.5$ hours. The delay to operation exceeded 24 hours in 2 (4.3\%) cases due to misdiagnosis.

\section{Pathological Data}

In our cohort, $52(54.2 \%)$ of the elderly patients had complicated appendicitis (Table 2). Only one person (1\%) had a benign tumor-a polyp. Interestingly, two cases of complicated appendicitis were identified as stump appendicitis. In one of these cases, the patient had undergone laparoscopic appendectomy three years 
TABLE 2. Types of Acute Appendicitis in Our Study Population

\begin{tabular}{ccc}
\hline \multicolumn{2}{c}{ Pathologic Description of Acute Appendicitis } & Entire Cohort $(\mathrm{n}=96)$ \\
\hline Uncomplicated & Inflamed & $6(6.25 \%)$ \\
& Suppurative & $38(39.6 \%)$ \\
Complicated & Fecalith present & $22(22.7 \%)$ \\
& Perforated & $48(50 \%)$ \\
& Gangrenous & $4(4.2 \%)$ \\
& Fecalith present & $30(30.8 \%)$ \\
\hline
\end{tabular}

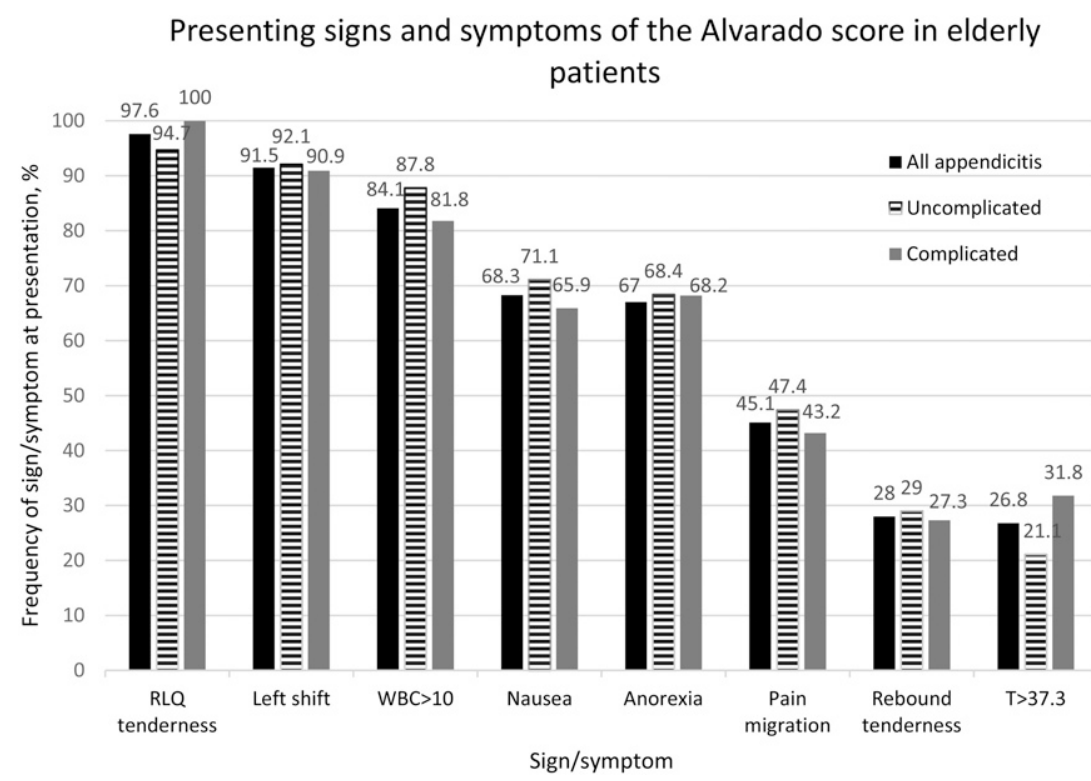

FIG. 1. Presenting signs and symptoms of the Alvarado score in elderly patients.

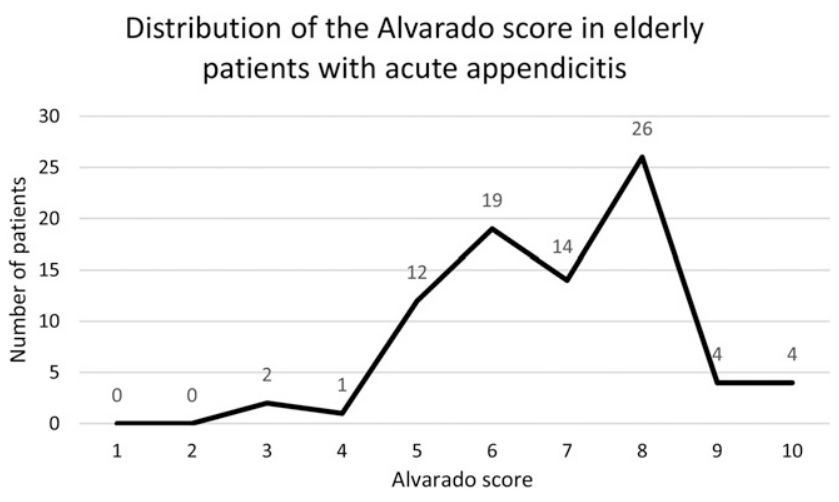

FIG. 2. Distribution of the Alvarado score in elderly patients with acute appendicitis.

previously; in the other case, there was an accidental appendectomy during a $\mathrm{C}$-section many years prior.

\section{Presenting Symptom Characteristics}

Tenderness in the RLQ, left shift of neutrophils (neutrophilia $\geq 70 \%$ ), and leukocytosis (WBC $>10$ ) were more common $(97.6 \%, 91.5 \%$, and $84.1 \%$, respectively) for elderly patients in our study than other presenting symptoms and signs included in the
Alvarado scoring system (Fig. 1). ${ }^{4}$ The signs and symptoms with lowest correlation in elderly patients were migration of pain to the RLQ, presence of rebound tenderness, and temperature elevation to $\geq 37.3^{\circ} \mathrm{C}$ (Fig. 2).

\section{Distribution of the Alvarado Score}

The mean Alvarado Score in our study population was $6.9 \pm 0.33$ for patients who had the requisite variables to calculate the Alvarado score $(n=82)$ in the entire cohort. The frequency of low-risk Alvarado scores (1-4) was observed in 3.7 per cent of cases. Alvarado scores indicating intermediate risk of appendicitis (5-8) were observed in 86.6 per cent of cases and high-risk scores of 9 or 10 were observed in 9.7 per cent of cases. An Alvarado score between 5 and 8 was found to correlate more for acute appendicitis in our elderly cohort, irrespective of the pathological type of appendicitis.

Comparative analysis of our data in the elderly population with the original Alvarado data is shown in Table 3. There was a statistically significant increase in the number of patients scoring 5 or 6 in our cohort compared with the original Alvarado cohort $(P<0.01)$; 
TABLE 3. The Comparison of Distribution of Alvarado Scores in Our Study with Original Alvarado Data

\begin{tabular}{lccc}
\hline Score Distribution & Our Data $(\%)$ & Original Alvarado Data ${ }^{4}(\%)$ & P Value \\
\hline Score 1-4 & 3.7 & 3.5 & 0.92 \\
Score 5-6 & 37.8 & 15.4 & $<0.001$ \\
Score 7-10 & 58.5 & 81 & 0.01 \\
Score 7-8 & 48.8 & 48.9 & 0.99 \\
Score 9-10 & 9.7 & 32.1 & $<0.001$ \\
\hline
\end{tabular}

these scores were observed in elderly patients 2.5 times more frequently.

\section{Postoperative Course}

Thirty-one $(33 \%)$ patients suffered a total of 38 postoperative complications, as outlined in Table 4. An image guided drain was placed in nine patients with intra-abdominal abscesses. A total of five $(5.3 \%)$ patients underwent reoperation secondary to small bowel obstruction, wound dehiscence, or ileostomy takedown. In patients with complicated appendicitis, intraabdominal abscesses and reoperations were more common. However, wound infection rates were similar in both groups.

The overall mean length of stay in the hospital is $5.88 \pm 1.02$ days. Patients with uncomplicated and complicated appendicitis stayed in the hospital an average of 4.3 versus 7.1 days $(P=0.005)$. A majority of patients were discharged to their prior residential situation (home versus skilled nursing facility). Only 16 patients $(19 \%)$ temporarily lost independence and required home health services, whereas 4 patients $(4.8 \%)$ permanently lost their ability of living independently and required placement in a skilled nursing facility. Table 5 demonstrates discharge destinations for the study population. The postoperative mortality rate was 1.2 per cent; one patient with complicated appendicitis and several significant comorbidities, including metastatic lung cancer, coronary artery disease, and history of deep vein thrombosis, died.

\section{Discussion}

In this study, we show that the use of the Alvarado scoring system for the diagnosis of acute appendicitis requires modification to maintain reliability in patients 65 years of age and older. Studies have shown that as our nation's population continues to age, the number of elderly patients presenting with acute abdominal pain will increase. Similarly, a commensurate increase in the number of elderly patients diagnosed with and treated for acute appendicitis must be anticipated. ${ }^{6}$ Numerous studies have demonstrated a delay in presentation, increased risk of perforation and associated complications, as well as increased morbidity and mortality in this aged population. ${ }^{2,7}$ Our data further
TABLe 4. Frequency of Postoperative Complications

\begin{tabular}{lc}
\hline \multicolumn{1}{c}{ Postoperative Complication } & Number $(\%)$ \\
\hline Wound infection & $12(12.8)$ \\
Intra-abdominal abscess & $10(10.6)$ \\
Small bowel obstruction & $4(4.3)$ \\
Wound hematoma & $2(2.1)$ \\
Urinary tract infection & $2(2.1)$ \\
Pulmonary edema & $2(2.1)$ \\
Wound dehiscence & $1(1)$ \\
Rectal bleeding (after ileocectomy) & $1(1)$ \\
Pneumonia & $1(1)$ \\
Pulmonary embolus & $1(1)$ \\
Myocardial infarction & $1(1)$ \\
Clostridium difficile colitis & $1(1)$
\end{tabular}

TABLE 5. Discharge Destinations of Elderly Patients after Appendectomy

\begin{tabular}{lc}
\hline \multicolumn{1}{c}{ Discharge Destination } & Number $(\%)$ \\
\hline Home with no services & $60(71.4)$ \\
$\begin{array}{l}\text { Home with home health care } \\
\text { Skilled nursing facility, where } \\
\text { patient previously resided }\end{array}$ & $16(19)$ \\
$\begin{array}{l}\text { Skilled nursing facility, new } \\
\text { placement }\end{array}$ & $3(3.6)$ \\
$\begin{array}{l}\text { Acute inpatient rehabilitation } \\
\text { Long term acute care hospital }\end{array}$ & $3(3.6)$ \\
Death in hospital & $1(1.2)$ \\
\end{tabular}

support these findings and clearly identify the need for improved and timely diagnosis of acute appendicitis in the elderly.

The rate of complicated appendicitis in the elderly was higher in our study than is reported in the general population, 50 versus 20 to 30 per cent, respectively. ${ }^{2,4,8-10}$ More than 10 per cent of elderly patients in our study presented more than once to the ER or clinic before appropriate diagnosis of appendicitis was made; perforation was identified in all of these patients at the time of operation. Other authors report similar delays in elderly. ${ }^{11,12}$

Symptoms that demonstrated a stronger association with acute appendicitis in our study included tenderness in the RLQ, leukocytosis, and presence of left shift (neutrophilia $\geq 70 \%$ ). Other studies have demonstrated that one of the greatest predictors of acute appendicitis are laboratory inflammatory symptoms and, in opposition to our results, pain migration and peritoneal irritation signs. ${ }^{13}$ Unfortunately, the diagnostic specificity 
of these symptoms remains low. ${ }^{4}$ Temperature elevation and rebound tenderness were not reported in nearly 70 per cent of elderly patients in our study at time of presentation. These findings may be due to age-related relative immunomodulation ${ }^{14}$ and weakness of the abdominal wall. ${ }^{15}$ However, as previously shown by meta-analysis, variances of the Alvarado score are weak individual predictors, but in combination provide high discriminating power. ${ }^{13,16}$

Length of stay in our study (5.9 days) was comparable to data from other studies (4.8-7.8 days). ${ }^{9}, 10$ The overall morbidity rate of 33 per cent in our study was in line with numerous other publications, ${ }^{7,17,18}$ but it was higher than some reported data. ${ }^{2,11}$ The mortality rate in our study (1\%) was similar to other recent studies in the elderly population. ${ }^{9-11,18}$

Although the preponderance of females in our study is unlike the demographics of the younger population, this ratio is consistent with the findings of other authors, $, 9,17,19,20$ and could be related to the longer life expectancy of women. Sensitivity of CT in diagnosis of acute appendicitis among elderly patients was lower than in literature for the general population $(81.6 \% \mathrm{vs}$ 95-100\%), ${ }^{21,22}$ likely linked to a low prevalence of appendicitis in the elderly population. Delay in operation for more than 24 hours was only seen in 4.3 per cent of cases, which was lower than seen in other studies $(13-21 \%)^{2,}, 18$

Some authors have suggested that the use of the Alvarado score portends a misdiagnosis of appendicitis in elderly patients ${ }^{21,23}$ or recommends use of the Alvarado score to only "rule out" appendicitis for scores of 5 or less. ${ }^{24}$ Our analysis suggests that modifying the clinical interpretation of the Alvarado score may result in an appropriate and more timely diagnosis of appendicitis in this high-risk population. On the basis of our data, the vast majority of elderly patients with pathologically confirmed appendicitis (86.6\%) had an Alvarado score ranging from 5 to 8, with nearly half (40\%) scoring either 5 or 6 . Therefore, nearly half of the elderly patients presented with an intermediate index of suspicion for appendicitis based on current Alvarado score interpretation. Our study demonstrates that using the current interpretation of the Alvarado score in elderly patients would underestimate those with acute appendicitis, leading to increased risk of misdiagnosis and associated delays in care with resultant increased morbidity and mortality. To maintain utility of the scoring system for this growing population, our data suggest that interpretation of Alvarado score should be modified (see Table 6). According to our data, Alvarado scores ranging from 5 to 10 should correspond to high risk of appendicitis in the elderly.
TABLE 6. Proposed Changes in the Interpretation of the Alvarado Score for Elderly

$$
\begin{array}{ll}
\text { Alvarado Score } & \\
1-4=\text { low risk } & \text { Our Modified Interpretation } \\
5-6=\text { intermediate risk } & 5-4=\text { low risk } \\
7-10=\text { high risk } &
\end{array}
$$

This study is limited by its retrospective nature and inherent inability to exclude biases, such as selection, presentation, or pathology bias. The population also represents a cohort from a single institution, which may harbor inherent selection bias. Additionally, our data set excludes patients who did not undergo surgical treatment of appendicitis, such as those treated conservatively with antibiotics alone or those who underwent a radiologic drainage procedure for abscess with subsequent interval appendectomy. However, our study contains a large number of elderly patients with acute appendicitis in comparison to prior studies, and a majority of data were collected from objective sources.

\section{Conclusion}

Physicians should have a higher clinical suspicion of acute appendicitis in elderly patients who present with RLQ tenderness, leukocytosis, or presence of left shift, and should not rule out appendicitis in the absence of fever or peritoneal signs. Most importantly, this study suggests that altering our interpretation of the Alvarado score to classify elderly patients who present with a score of 5 or greater as high risk may lead to more timely diagnosis of acute appendicitis. Prospective studies are needed to validate our findings.

\section{REFERENCES}

1. Horattas MC, Guyton DP, Wu D. A reappraisal of appendicitis in the elderly. Am J Surg 1990;160:291-3.

2. Storm-Dickerson TL, Horattas MC. What have we learned over the past 20 years about appendicitis in the elderly? Am J Surg 2003;185:198-201.

3. Franz MG, Norman J, Fabri PJ. Increased morbidity of appendicitis with advancing age. Am Surg 1995;61:40-4.

4. Alvarado A. A practical score for the early diagnosis of acute appendicitis. Ann Emerg Med 1986;15:557-64.

5. Andersson M, Andersson RE. The appendicitis inflammatory response score: a tool for the diagnosis of acute appendicitis that outperforms the Alvarado score. World J Surg 2008;32:1843-9.

6. Buckius MT, McGrath B, Monk J, et al. Changing epidemiology of acute appendicitis in the United States: study period 19932008. J Surg Res 2012;175:185-90.

7. Gurleyik G, Gurleyik E. Age-related clinical features in older patients with acute appendicitis. Eur J Emerg Med 2003;10:200-3.

8. Schwartz SI. Principles of Surgery, 6th Ed. New York, NY: McGraw Hill, Health Professions Division, 1994. 
9. Harbrecht BG, Franklin GA, Miller FB, et al. Acute appendicitisnot just for the young. Am J Surg 2011;202:286-90.

10. Korner H, Sondenaa K, Soreide JA, et al. Incidence of acute nonperforated and perforated appendicitis: age-specific and sexspecific analysis. World J Surg 1997;21:313-7.

11. Bhullar JS, Chaudhary S, Cozacov Y, et al. Acute appendicitis in the elderly: Diagnosis and management still a challenge. Am Surg 2014;80:E295-7.

12. Ghnnam WM. Elderly versus young patients with appendicitis 3 years experience. Alexandria J Med 2012;48:9-12.

13. Andersson RE. Meta-analysis of the clinical and laboratory diagnosis of appendicitis. Br J Surg 2004;91:28-37.

14. Podnos YD, Jimenez JC, Wilson SE. Intra-abdominal sepsis in elderly persons. Clin Infect Dis 2002;35:62-8.

15. Macaluso CR, McNamara RM. Evaluation and management of acute abdominal pain in the emergency department. Int J Gen Med 2012;5:789-97.

16. Wray CJ, Kao LS, Millas SG, et al. Acute appendicitis: controversies in diagnosis and management. Curr Probl Surg 2013; 50:54-86.
17. Lee JF, Leow CK, Lau WY. Appendicitis in the elderly. Aust N Z J Surg 2000;70:593-6.

18. Hui TT, Major KM, Avital I, et al. Outcome of elderly patients with appendicitis: effect of computed tomography and laparoscopy. Arch Surg 2002;137:995-8, discussion 9-1000.

19. Blomqvist PG, Andersson RE, Granath F, et al. Mortality after appendectomy in Sweden, 1987-1996. Ann Surg 2001;233:455-60.

20. Addiss DG, Shaffer N, Fowler BS, et al. The epidemiology of appendicitis and appendectomy in the United States. Am J Epidemiol 1990;132:910-25.

21. Gwynn LK. The diagnosis of acute appendicitis: clinical assessment versus computed tomography evaluation. J Emerg Med 2001;21:119-23.

22. Dahabreh IJ, Adam GP, Halladay CW, et al. Diagnosis of Right Lower Quadrant Pain and Suspected Acute Appendicitis. Rockville, MD: AHRQ Comparative Effectiveness Reviews, 2015.

23. Segev L, Keidar A, Schrier I, et al. Acute appendicitis in the elderly in the twenty-first century. J Gastrointest Surg 2015;19:730-5.

24. Ohle R, O'Reilly F, O'Brien KK, et al. The Alvarado score for predicting acute appendicitis: a systematic review. BMC Med 2011;9:139. 